\title{
Prevalence and Risk Factors of Type 2 Diabetes and Prediabetes Among 53,288 Middle-Aged and Elderly Adults in China: A Cross-Sectional Study
}

\author{
Mengdi Xia ${ }^{1, *}$ \\ Kaixiang $\mathrm{Liu}^{2, *}$ \\ Jie Feng' \\ Zaiqiong Zheng' \\ Xisheng Xie (D)
}

'Department of Nephrology, The Second Clinical Medical Institution of North Sichuan Medical College (Nanchong Central Hospital) and Nanchong Key Laboratory of Basic Science \& Clinical Research on Chronic Kidney Disease, Nanchong, Sichuan, People's Republic of China; ${ }^{2}$ Department of Nephrology, Sichuan Provincial People's Hospital, School of Medicine, University of Electronic Science and Technology of China, Chengdu, Sichuan, People's Republic of China

*These authors contributed equally to this work

Correspondence: Xisheng Xie Department of Nephrology, The Second Clinical Medical Institution of North Sichuan Medical College (Nanchong Central Hospital), South Renmin Road 97, Shunqing District, Nanchong, Sichuan Province, 637000, People's Republic of China

Tel +86I58 8I70 0265

Email xishengx@I26.com
Background: Diabetes is a metabolic disorder that causes a heavy burden on healthcare systems worldwide. The aim of this study was to determine the prevalence of type 2 diabetes and prediabetes and its associated factors among eight communities in Nanchong, China.

Methods: This was an observational cross-sectional study conducted throughout eight communities in Nanchong, China. The participants were 53,288 individuals aged 45 years or older. The participants' characteristics, comorbidities, health behaviors, family history, and dietary intake were assessed. Multinomial logistic regression models were fitted to identify factors associated with type 2 diabetes and prediabetes.

Results: The prevalence of diabetes and prediabetes was $13.9 \%$ (95\% confidence interval [CI], 13.6-14.2) and 3.1\% (95\% CI, 2.9-3.2) of the population, respectively. After adjusting for other risk factors, advanced age, overweight, obesity, abdominal obesity, comorbidities, smoking, a family history of diabetes, and Chinese cooking vegetable intake were associated with an increased risk of type 2 diabetes and prediabetes.

Conclusion: The prevalence of type 2 diabetes in the Chinese population is rising compared with data from the past. The risk factors of type 2 diabetes and prediabetes identified in this study will aid the identification of individuals at a high-risk of diabetes and the implementation of effective health promotion programs and campaigns.

Clinical Trial Registry Number: ChiCTR-HOC-17013200.

Keywords: cross-sectional study, diabetes, prevalence, risk factors

\section{Introduction}

The prevalence of diabetes is increasing worldwide. In 2019, more than 463 million people had diabetes, and the incidence is projected to increase to 700 million by $2045 .{ }^{1}$ Over $90 \%$ of persons with diabetes have type 2 diabetes mellitus (T2DM). ${ }^{2}$ T2DM is associated with numerous complications, including cardiovascular disease (CHD), peripheral neuropathy, stroke, infection, chronic renal failure, and retinopathy. All of these complications significantly contribute to the high mortality, morbidity, and socioeconomic burden associated with diabetes. ${ }^{3}$ There are no disease-modifying interventions for T2DM and its complications. Therefore, the disease can only be controlled through medication and lifestyle changes, ${ }^{4}$ resulting in a heavy economic burden for patients and healthcare systems. ${ }^{5}$

Diabetes, particularly T2DM, has become a major public health issue in China and has become a relevant socioeconomic burden. ${ }^{2}$ However, although the prevalence and risk factors of diabetes have been studied in China, studies on the risk 
factors and the trend of prediabetes, particularly among middle-aged and elderly adults, are scarce. ${ }^{6}$ China has a vast territory characterized by lifestyle diversity and marked differences in economic and social development endeavors among its regions. Therefore, the diabetes prevalence greatly varies between China's regions. ${ }^{7}$ Nanchong is an important transportation hub and representative city in western China. The population of this city has a recognizable age imbalance, given the high proportion of older people, in line with the country's rapidly aging population. T2DM mainly affects middle-aged and elderly people, accounting for more than $90 \%$ of the diabetes cases. ${ }^{8}$ The rapidly aging population, the rapid increase in the incidence of chronic diseases, and epidemiological changes will put significant financial pressure on healthcare systems in the future. ${ }^{9}$ Given that our knowledge of the status of diabetes and prediabetes among the elderly in the urban areas of western China is limited, this study aimed to explore the incidence and risk factors of $\mathrm{T} 2 \mathrm{DM}$ and prediabetes in the Nanchong region.

\section{Methods}

\section{Study Design and Setting}

This observational and cross-sectional study was conducted between November 2012 and December 2017. The participants were recruited from eight communities in the Shunqing District, Nanchong City, China. The communities involved were Xin Jian, Dongnan, Huafeng, Wufeng, Beicheng, Heping Road, Dongcheng, and Zhongcheng. The numbers of households in the eight communities were used as labels, and 1,000 labels were randomly selected from each community via a computer random number generator for questionnaire surveys. The unified quality control plan was formulated by the Center for Disease Control and Prevention of Nanchong and the Center for Comprehensive Management of Chronic Diseases of Nanchong Central Hospital.

\section{Ethical Aspects}

This study was approved by the Medical Ethics Committee of Nanchong Central Hospital (2012-65) and registered in the China Clinical Trial Registry (NO: ChiCTR-HOC -17013200). All procedures met the institution's ethical standards and were conducted in compliance with the International Declaration of Helsinki. Written informed consent was obtained from all participants.

\section{Study Population}

A total of 105,956 individuals older than 45 years were included in the survey. From this population, we excluded 50,294 individuals who were previously diagnosed with type 1 diabetes or other types of diabetes $(n=344)$, and persons who had never taken blood-glucose measurements or were unsure whether they had diabetes $(n=49,950)$. Additionally, 2,374 individuals with missing values in any variables were also excluded. Finally, 53,288 individuals aged between 45 years and 101 years were included in the analysis. Of them, 7,396, 1,643, and 44,249 individuals had T2DM, prediabetes, and no diabetes, respectively (Figure 1). T2DM, prediabetes, and non-diabetes were defined following the World Health Organization criteria. $^{10}$

\section{Data Collection}

Data on the participants' characteristics were collected from the questionnaire and clinical examination records. Age, sex, education, income, health behaviors, comorbidities, family history of diabetes, and dietary intake were self-reported during face-to-face interviews. Physical examinations included weight, height, and waist circumference. Well-documented fasting serum glucose measurements obtained during the outpatient visit were recorded.

\section{Definitions}

Anthropometric variables included height, weight, and waist circumference and were measured by medical staff members. Body mass index (BMI) was classified into normal weight $\left(<23.0 \mathrm{~kg} / \mathrm{m}^{2}\right)$, overweight $(23-27.5 \mathrm{~kg} /$ $\left.\mathrm{m}^{2}\right)$, and obese $\left(\geq 27.5 \mathrm{~kg} / \mathrm{m}^{2}\right)$ following the WHO classification and BMI recommendations for Asian populations. ${ }^{11}$ Waist circumference was recorded either as normal $(<85 \mathrm{~cm}$ in women and $<90 \mathrm{~cm}$ in men) or indicative of abdominal obesity $(\geq 85 \mathrm{~cm}$ in women and $\geq$ $90 \mathrm{~cm}$ in men). ${ }^{12}$

The educational categories used in this study were low (primary and lower), middle (middle and high school), and high (college and above). Household income was classified (based on the per capita annual income in China) into poor $[<¥ 10,000(1496 \$) /$ year], middle class $[¥ 10,000$ 20,000 (1496-2986 \$)/year], well-off $[¥ 20,000-50,000$ (2986-7467 \$)/year], and rich[> ¥50,000 (7467 \$)/year)].

Hypertension and hyperlipidemia were defined based on a previous diagnosis. CHD was determined based on the presence or absence of coronary atherosclerotic heart 


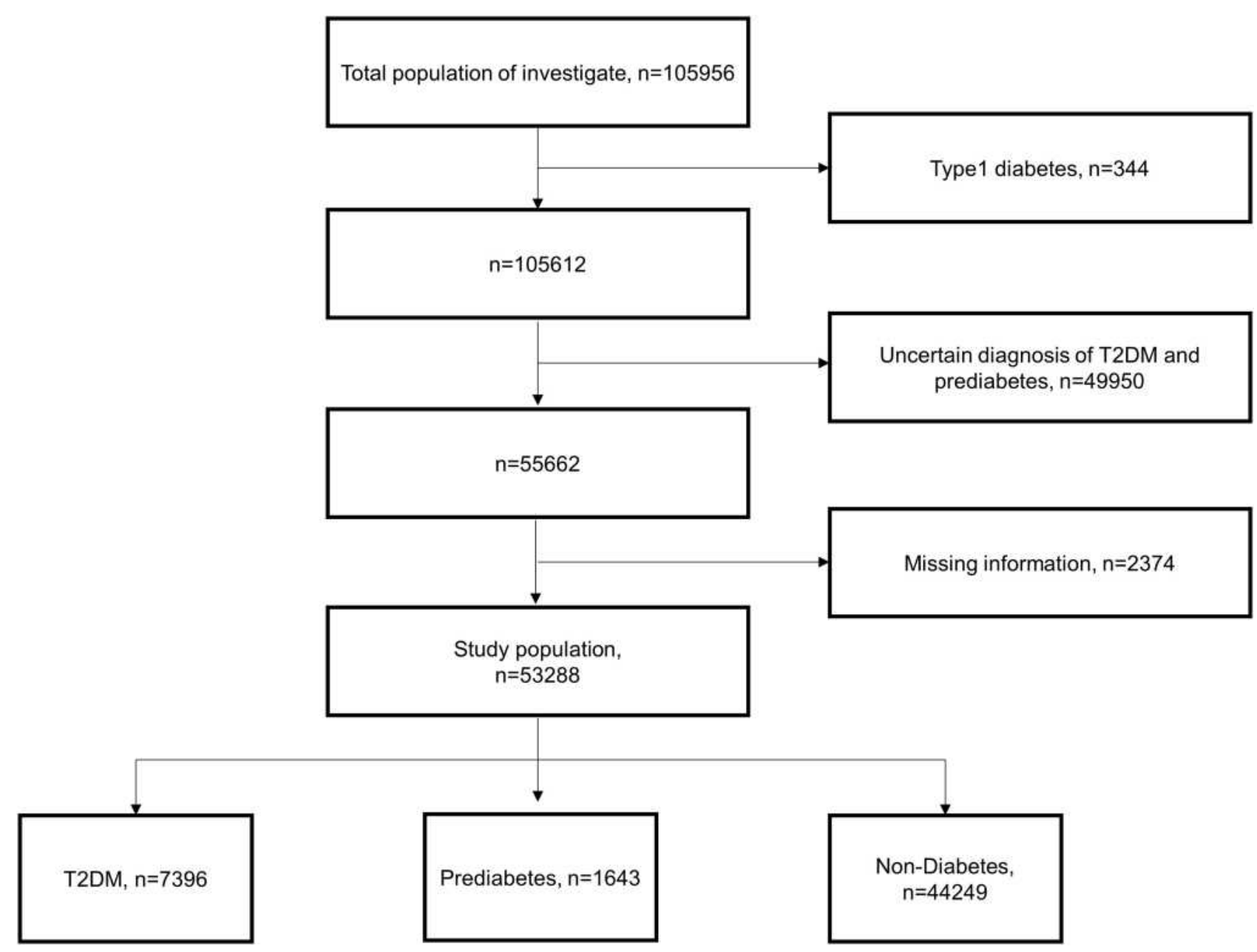

Figure I Flow chart documenting inclusion criteria, exclusion criteria, and the number of individuals included in the study population.

disease. Stroke was defined as having a recorded diagnosis of cerebral hemorrhage, cerebral infarction, or other cerebrovascular diseases. A family history of diabetes was determined as having a relative within three generations or multiple family members being diagnosed with diabetes.

Smoking status was classified into smokers (at least one cigarette per day), ex-smokers (people who quit smoking $>1$ year ago at the time of the study), and non-smokers (those who never smoked). ${ }^{13}$ Alcohol intake was categorized into non-drinkers ( $0 \mathrm{~g}$ alcohol/day), light (1$14.99 \mathrm{~g} /$ day), moderate (15-29.99 g/day), and heavy drinkers ( $\geq 30 \mathrm{~g} /$ day). ${ }^{14,15}$

We used a questionnaire similar to the International Physical Activity Questionnaire ${ }^{16}$ and collected the cumulative hours per day spent at work, transportation, housework, and leisure physical activity in the past seven days. The physical activity level, based on metabolic equivalents of task score (METs), was divided into low, medium, and high, following international physical activity standards. ${ }^{17}$
Sleep was assessed using the Pittsburgh Sleep Quality Index Scale. ${ }^{18}$ Sleep status was divided into three categories: $\leq 5 \mathrm{~h}, 6-9 \mathrm{~h}$, and $>9 \mathrm{~h}^{19}$ Considering the actual status of the Chinese community diet, the participants' current dietary intake was evaluated as follows: meat or poultry intake, $<100 \mathrm{~g} /$ day or $>100 \mathrm{~g} /$ day; vegetable intake, $100 \mathrm{~g} /$ day or $>100 \mathrm{~g} /$ day; added fish consumption, yes or no; soy, yes or no; dairy product, yes or no; and fruits, yes or no.

\section{Statistical Analyses}

The overall prevalence of T2DM and its associated factors were determined and recorded using the descriptive and inferential method of data analysis. Descriptive statistics were used to summarize the characteristics of study participants. Continuous variables (age, BMI, waist circumference, sleep, and MET) were expressed as the mean \pm standard deviation (SD) and compared using an ANOVA when variables were normally distributed. For variables that did not follow 
a normal distribution, a Kruskal-Wallis test was used. Descriptive statistics of categorical variables were presented as frequency and percentage and analyzed using the Chisquared test. Multivariate logistic analysis was used to assess the association between the explanatory variables and the outcome variable to control the effect of confounding and the independent effect of each variable on diabetes and prediabetes. These data are presented as odds ratios (ORs) and a 95\% confidence interval (CI). All statistical analyses were performed using the IBM SPSS Version 20.0 (IBM Corp, Armonk, NY, USA). A $p$-value of $<0.05$ was considered statistically significant.

\section{Results}

The average age of the study population was $63.7 \pm 10.0$ (mean $\pm \mathrm{SD}$ ) years and was similar in three groups. The mean BMI of the overall study population was $24.2 \pm$ $3.2 \mathrm{~kg} / \mathrm{m}^{2}$, and the average WC was $82.6 \pm 8.6 \mathrm{~cm}$. The average sleep duration was $7.0 \pm 1.1$ hours (Table 1). In total, $13.9 \%(95 \% \mathrm{CI}, 13.6-14.2)$ and $3.1 \%(95 \% \mathrm{CI}$, 2.9-3.2) had T2DM and prediabetes, respectively. In all three groups, the majority of the participants were female, had moderate-level income, and had primary and middlelevel education. Most of the participants in the T2DM group were older than 65 years. Nearly $30 \%$ of the participants had comorbidities, and most participants had hypertension and hyperlipidemia. More than $10 \%$ of the participants were either smokers or drinkers, or both. More than $90 \%$ of the participants had moderate physical activity levels, and few had high physical activity levels. There were no significant differences in this regard between the three groups. Furthermore, the three groups also showed similar sleep habits, with most of the participants having a sleep duration of 6-9 h, few slept $\leq 6 \mathrm{~h}$, and very few slept $>9 \mathrm{~h}$. Dietary habits were similar in the three groups and showed a trend of low intake of protein-rich foods such as fish, meat/poultry, and soybeans. Over $90 \%$ of the participants hardly consumed fish, soybeans, and meat $(<$ $100 \mathrm{~g}$ per day).

In all three groups, the proportion of participants with T2DM significantly differed according to age; sex; BMI; abdominal obesity; education; income; comorbidities like hypertension, hyperlipidemia, CHD and stroke; smoking; drinking; sleep; family history of diabetes; and dietary intake of fish, vegetables, soy, dairy product, and fruits (Table 2).

Age, sex, education, income, comorbidities, smoking, drinking, sleep duration, family history of diabetes, BMI, and abdominal obesity were included in the multivariate regression analysis. After adjusting for other factors, the result showed that compared with people younger than 65 years, participants aged $65-80$ years $(p<0.001)$ and older than $80(p<0.001)$ had higher odds of having diabetes and prediabetes $(p=0.044, p<0.001$, respectively). Individuals who were overweight and obese also had higher odds of T2DM and prediabetes than those with normal BMI $(p<0.001)$. Furthermore, abdominally obese people had higher odds of diabetes and prediabetes $(p<0.001)$ than persons with normal abdominal girth (Table 3).

The risk of T2DM was significantly higher in individuals with lower education, comorbidities like hypertension and hyperlipidemia, CHD and stroke, who were smokers, with short sleep duration, a family history of diabetes, consumed dairy products, had a vegetable intake $\geq 100 \mathrm{~g}$, and had no soy and fruit intake. The significant findings in the prediabetes group were also observed in the diabetes group. However, smoking, short sleep, drinking, and comorbidities did not increase the risk of prediabetes, unlike higher education, which increased the risk of prediabetes. (Table 3).

\section{Discussion}

Data on diabetes and prediabetes among middle-aged and elderly adults in the urban areas of western China are

Table I The Continuous Variables of Participants. (Mean \pm SD)

\begin{tabular}{|l|c|c|c|c|}
\hline Variables & Total & T2DM & Prediabetes & Non-Diabetes \\
\hline Age & $63.7 \pm 10.0$ & $67.2 \pm 9.3$ & $63.9 \pm 9.9$ & $63.7 \pm 10.0$ \\
BMI $\left(\mathrm{kg} / \mathrm{m}^{2}\right)$ & $24.2 \pm 3.2$ & $24.6 \pm 3.4$ & $24.6 \pm 3.1$ & $24.1 \pm 3.2$ \\
Waist circumference $(\mathrm{cm})$ & $82.6 \pm 8.6$ & $84.6 \pm 10.0$ & $83.5 \pm 8.3$ & $82.2 \pm 8.3$ \\
Sleep duration (hour) & $7.0 \pm 1.1$ & $6.9 \pm 1.2$ & $7.1 \pm 1.1$ & $7.0 \pm 1.0$ \\
METs & $1500.1 \pm 571.5$ & $1475.4 \pm 568.8$ & $1484.6 \pm 572.4$ & $1504.8 \pm 571.8$ \\
\hline
\end{tabular}

Abbreviations: BMI, body mass index; METs, metabolic equivalent of task score. 
Table 2 Characteristics of the 53,288 Respondents Included in This Study

\begin{tabular}{|c|c|c|c|c|c|}
\hline \multirow[t]{2}{*}{ Characteristics } & \multirow[t]{2}{*}{ Total } & \multicolumn{4}{|c|}{ Frequency (\%) } \\
\hline & & T2DM & Prediabetes & Non-Diabetes & $P$ value \\
\hline \multicolumn{6}{|l|}{ Age (Year) } \\
\hline$<65$ & $29,102(54.6)$ & 2880 (38.9) & $854(52.0)$ & $25,368(57.3)$ & $<0.001$ \\
\hline $65-80$ & $20,517(38.5)$ & $3758(50.8)$ & $694(42.2)$ & 16,065 & \\
\hline$\geq 80$ & $3669(6.9)$ & $758(10.2)$ & $95(5.8)$ & $2816(6.4)$ & \\
\hline \multicolumn{6}{|l|}{ Gender } \\
\hline Male & $20,024(37.6)$ & $3081(41.7)$ & 656 (39.9) & 16,287 (36.8) & $<0.001$ \\
\hline Female & $33,264(62.4)$ & $4315(58.3)$ & $987(60.1)$ & $27,962(63.2)$ & \\
\hline \multicolumn{6}{|l|}{ BMI } \\
\hline$<23$ & $19,308(36.2)$ & $2322(31.4)$ & $482(29.3)$ & $16,504(37.3)$ & $<0.001$ \\
\hline $23-27.5$ & $26,877(50.4)$ & $3862(52.2)$ & $906(55.1)$ & $22,109(50.0)$ & \\
\hline$\geq 27.5$ & $7103(13.3)$ & $1212(16.4)$ & $255(15.5)$ & $5636(12.7)$ & \\
\hline \multicolumn{6}{|l|}{ Waist circumference $\dagger$} \\
\hline Normal & $38,674(72.6)$ & $4672(63.2)$ & $1130(68.8)$ & $32,872(74.3)$ & $<0.001$ \\
\hline Abdominal obesity & $|4,6| 4(27.4)$ & $2724(36.8)$ & $5 \mid 3(3 \mid .2)$ & II,377 (25.7) & \\
\hline \multicolumn{6}{|l|}{ Education } \\
\hline Primary/under & $23,877(44.8)$ & $3804(51.4)$ & $574(34.9)$ & $19,499(44.1)$ & $<0.001$ \\
\hline Middle and High & $26,077(48.9)$ & $3094(41.8)$ & $94 \mid(57.3)$ & $22,042(49.8)$ & \\
\hline College/above & $3334(6.3)$ & $498(6.7)$ & $128(7.8)$ & $2708(6.1)$ & \\
\hline \multicolumn{6}{|l|}{ Household income } \\
\hline Poor & $290(0.5)$ & $48(0.6)$ & $12(0.7)$ & $230(0.5)$ & 0.024 \\
\hline Moderate & $47,269(88.7)$ & $6618(89.5)$ & I 449 (88.2) & $39,202(88.6)$ & \\
\hline Well-off & $5663(10.6)$ & $723(9.8)$ & I82 (II.I) & $4758(10.8)$ & \\
\hline Rich & $66(0.1)$ & $7(0.1)$ & $0(0) \ddagger$ & $59(0.1)$ & \\
\hline \multicolumn{6}{|l|}{ Comorbidities } \\
\hline \multicolumn{6}{|l|}{ Hypertension } \\
\hline No & $37,816(71.0)$ & $4506(60.9)$ & II 27 (68.6) & $32,183(72.7)$ & $<0.001$ \\
\hline Yes & $15,472(29.0)$ & $2890(39.1)$ & $516(31.4)$ & $12,066(27.3)$ & \\
\hline \multicolumn{6}{|l|}{ Hyperlipidemia } \\
\hline No & $49,694(93.3)$ & $6337(85.7)$ & I 367 (83.2) & $41,990(94.9)$ & $<0.001$ \\
\hline Yes & $3594(6.7)$ & $1059(14.3)$ & $276(16.8)$ & $2259(5.1)$ & \\
\hline \multicolumn{6}{|l|}{$\mathrm{CHD}$} \\
\hline No & $52,606(98.7)$ & $7194(97.3)$ & $1617(98.4)$ & 43,795 (99.0) & $<0.001$ \\
\hline Yes & $682(1.3)$ & $202(2.7)$ & $26(1.6)$ & $454(1.0)$ & \\
\hline \multicolumn{6}{|l|}{ Stroke } \\
\hline No & $52,643(98.8)$ & 7189 (97.2) & $1624(98.8)$ & $43,830(99.1)$ & $<0.001$ \\
\hline Yes & $645(1.2)$ & $207(2.8)$ & $19(1.2)$ & $419(0.9)$ & \\
\hline \multicolumn{6}{|l|}{ Health behaviors } \\
\hline \multicolumn{6}{|l|}{ Smoking } \\
\hline Non-smoker & $46,85 I(87.9)$ & $6396(86.4)$ & |43| (87.I) & $39,024(88.2)$ & 0.001 \\
\hline Ex-smoker & $1075(2.0)$ & $161(2.2)$ & $37(2.3)$ & $877(2.0)$ & \\
\hline Smoker & $5362(10.1)$ & $839(11.3)$ & $175(10.7)$ & $4348(9.8)$ & \\
\hline
\end{tabular}

(Continued) 
Table 2 (Continued).

\begin{tabular}{|c|c|c|c|c|c|}
\hline \multirow[t]{2}{*}{ Characteristics } & \multirow[t]{2}{*}{ Total } & \multicolumn{4}{|c|}{ Frequency (\%) } \\
\hline & & T2DM & Prediabetes & Non-Diabetes & $P$ value \\
\hline $\begin{array}{l}\text { Drinking } \\
\text { Non-drinkers } \\
\text { Light } \\
\text { Moderate } \\
\text { Heavy }\end{array}$ & $\begin{array}{c}14,893(27.9) \\
17,964(33.7) \\
13,192(24.7) \\
7239(13.6)\end{array}$ & $\begin{array}{l}2104(28.4) \\
2525(34.1) \\
1807(24.4) \\
960(13.0)\end{array}$ & $\begin{array}{l}445(27.1) \\
531(32.3) \\
394(24.0) \\
273(16.6)\end{array}$ & $\begin{array}{l}\mid 2,344(28.0) \\
\mid 4,908(33.7) \\
10,99 \mid(24.8) \\
6006(13.6)\end{array}$ & 0.012 \\
\hline $\begin{array}{l}\text { METs§ } \\
\text { Low } \\
\text { Medium } \\
\text { High }\end{array}$ & $\begin{array}{c}2308(4.3) \\
50,598(95.0) \\
382(0.7)\end{array}$ & $\begin{array}{c}346(4.7) \\
7002(94.7) \\
48(0.6)\end{array}$ & $\begin{array}{c}51(3.1) \\
1582(96.3) \\
10(0.6)\end{array}$ & $\begin{array}{c}1911(4.3) \\
42,014(94.9) \\
324(0.7)\end{array}$ & 0.060 \\
\hline $\begin{array}{l}\text { Sleep duration (Hour) } \\
\leq 5 \\
6-9 \\
>9\end{array}$ & $\begin{array}{c}3538(6.6) \\
49,294(92.5) \\
456(0.9)\end{array}$ & $\begin{array}{c}682(9.2) \\
6622(89.5) \\
92(1.2)\end{array}$ & $\begin{array}{c}129(7.9) \\
\text { I } 499(9 \mid .2) \\
15(0.9)\end{array}$ & $\begin{array}{c}2727(6.2) \\
4 I, 173(93.0) \\
349(0.8)\end{array}$ & $<0.001$ \\
\hline Family history & & & & & \\
\hline $\begin{array}{l}\text { Diabetes } \\
\text { No } \\
\text { Yes }\end{array}$ & $\begin{array}{c}50,218(94.2) \\
3070(5.8)\end{array}$ & $\begin{array}{l}6319(85.4) \\
1077(14.6)\end{array}$ & $\begin{array}{l}|47|(89.5) \\
\mid 72(10.5)\end{array}$ & $\begin{array}{c}42,428(95.9) \\
|82|(4.1)\end{array}$ & $<0.001$ \\
\hline Dietary intake & & & & & \\
\hline $\begin{array}{l}\text { Fish } \\
\qquad \begin{array}{l}\text { No } \\
\text { Yes }\end{array}\end{array}$ & $\begin{array}{c}51,368(96.4) \\
1920(3.6)\end{array}$ & $\begin{array}{c}7184(97.1) \\
212(2.9)\end{array}$ & $\begin{array}{c}1606(97.7) \\
37(2.3)\end{array}$ & $\begin{array}{c}42,578(96.2) \\
|67|(3.8)\end{array}$ & $<0.001$ \\
\hline $\begin{array}{l}\text { Meat/Poultry (g) } \\
\quad<100 \\
\quad \geq 100\end{array}$ & $\begin{array}{c}49,184(92.3) \\
4104(7.7)\end{array}$ & $\begin{array}{c}6859(92.7) \\
537(7.3)\end{array}$ & $\begin{array}{c}1517(92.3) \\
126(7.7)\end{array}$ & $\begin{array}{c}40,808(92.2) \\
344 I(7.8)\end{array}$ & 0.305 \\
\hline $\begin{array}{l}\text { Vegetables (g) } \\
\quad<100 \\
\geq 100\end{array}$ & $\begin{array}{l}26,030(48.9) \\
27,258(51.2)\end{array}$ & $\begin{array}{l}3503(47.3) \\
3893(52.6)\end{array}$ & $\begin{array}{l}660(40.2) \\
983(59.8)\end{array}$ & $\begin{array}{l}21,867(49.4) \\
22,382(50.6)\end{array}$ & $<0.001$ \\
\hline $\begin{array}{l}\text { Soy } \\
\text { No } \\
\text { Yes }\end{array}$ & $\begin{array}{c}49,037(92.0) \\
4251(8.0)\end{array}$ & $\begin{array}{c}6941(93.8) \\
455(6.2)\end{array}$ & $\begin{array}{c}|56|(95.0) \\
82(5.0)\end{array}$ & $\begin{array}{c}40,535(91.6) \\
3714(8.4)\end{array}$ & $<0.001$ \\
\hline $\begin{array}{l}\text { Dairy Product } \\
\text { No } \\
\text { Yes }\end{array}$ & $\begin{array}{l}38,83 \mid(72.9) \\
\mid 4,457(27.1)\end{array}$ & $\begin{array}{l}5110(69.1) \\
2286(30.9)\end{array}$ & $\begin{array}{l}1273(77.5) \\
370(22.5)\end{array}$ & $\begin{array}{l}32,448(73.3) \\
|I, 80|(26.7)\end{array}$ & $<0.001$ \\
\hline $\begin{array}{l}\text { Fruits } \\
\text { No } \\
\text { Yes }\end{array}$ & $\begin{array}{l}23,569(44.2) \\
29,719(55.8)\end{array}$ & $\begin{array}{l}3929(53.1) \\
3467(46.9)\end{array}$ & $\begin{array}{l}913(55.6) \\
730(44.4)\end{array}$ & $\begin{array}{l}18,727(42.3) \\
25,522(57.7)\end{array}$ & $<0.001$ \\
\hline
\end{tabular}

Notes: $\nmid$ Abdominal obesity: waist circumference $\geq 90 \mathrm{~cm}$ for men and $\geq 85 \mathrm{~cm}$ for women. $\ddagger$ The number of people in this group is zero and cannot be brought into the formula to calculate the percentage.

$\S$ Three levels of METs: low (any of the following two criteria: a. No activity reported; b. Some activities were reported, but they did not meet the medium criteria), medium (any of the following three criteria: a. Satisfy various high-intensity physical activities for at least 20 minutes per day, total $\geq 3 \mathrm{~d}$; b. Satisfy all kinds of medium-intensity or walking activities for at least 30 minutes every day, total $\geq 15 \mathrm{~d}$; c. Total physical activity of 3 intensities $\geq 5 \mathrm{~d}$, and total physical activity level of each week $\geq 600 \mathrm{MET}$-min/w), high (any of the following two criteria: a. various types of high-intensity physical activity total $\geq 3 \mathrm{~d}$, and the total weekly physical activity level $\geq 1500 \mathrm{MET}$-min/w; b. three types of physical activity total $\geq 7 \mathrm{~d}$, and the weekly total physical activity level $\geq 3000 \mathrm{MET}-\mathrm{min} / \mathrm{w}$ ).

Abbreviations: BMI, body mass index; METs, metabolic equivalent of task score; CHD, cardiovascular disease. 
Table 3 Participants Characteristics with or without Diabetes

\begin{tabular}{|c|c|c|c|c|c|c|c|c|}
\hline \multirow[t]{2}{*}{ Variables } & \multicolumn{4}{|c|}{ Crude OR (95\% Cl); $P$ value } & \multicolumn{4}{|c|}{ Adjusted OR† (95\% Cl); $P$ value } \\
\hline & T2DM & $\mathbf{P}$ & Prediabetes & $\boldsymbol{P}$ & T2DM & $\boldsymbol{P}$ & Prediabetes & $\mathbf{P}$ \\
\hline \multicolumn{9}{|l|}{ Age (Year) } \\
\hline$<65$ & Ref & & Ref & & Ref & & Ref & \\
\hline $65-80$ & $2.06(1.96-2.17)$ & $<0.001$ & $1.28(1.16-1.42)$ & $<0.001$ & $1.88(1.78-1.99)$ & $<0.001$ & $1.26(1.01-1.57)$ & 0.044 \\
\hline$\geq 80$ & $2.37(2.17-2.59)$ & $<0.001$ & $1.00(0.81-1.24)$ & 0.990 & $2.16(1.96-2.37)$ & $<0.001$ & $1.38(1.24-1.54)$ & $<0.001$ \\
\hline \multicolumn{9}{|l|}{ Gender } \\
\hline Male & $1.23(1.17-1.29)$ & $<0.001$ & $1.14(1.03-1.26)$ & 0.010 & $1.06(0.99-1.13)$ & 0.074 & $0.97(0.85-1.10)$ & 0.594 \\
\hline Female & Ref & & Ref & & Ref & & Ref & \\
\hline \multicolumn{9}{|l|}{ BMI } \\
\hline$<23$ & Ref & & Ref & & Ref & & Ref & \\
\hline $23-27.5$ & $1.24(1.18-1.31)$ & $<0.001$ & $1.40(1.25-1.57)$ & $<0.001$ & $1.59(1.47-1.72)$ & $<0.001$ & I.55 (I.33-I.8I) & $<0.001$ \\
\hline$\geq 27.5$ & $1.53(1.42-1.65)$ & $<0.001$ & $1.55(1.33-1.81)$ & $<0.001$ & $1.28(1.21-1.36)$ & $<0.001$ & $1.40(1.25-1.57)$ & $<0.001$ \\
\hline \multicolumn{9}{|l|}{ Waist circumference } \\
\hline Normal & Ref & & Ref & & Ref & & Ref & \\
\hline Abdominal obesity & $1.69(1.60-1.77)$ & $<0.001$ & $1.31(1.18-1.46)$ & $<0.001$ & $1.64(1.55-1.73)$ & $<0.001$ & $1.28(1.14-1.44)$ & $<0.001$ \\
\hline \multicolumn{9}{|l|}{ Education } \\
\hline Primary/under & Ref & & Ref & & Ref & & Ref & \\
\hline Middle and High & $0.72(0.68-0.76)$ & $<0.001$ & $1.45(1.32-1.95)$ & $<0.001$ & $0.82(0.78-0.87)$ & $<0.001$ & $1.53(\mid .38-1.7 I)$ & $<0.001$ \\
\hline College/above & $0.94(0.85-1.04)$ & 0.260 & $1.61(1.32-1.96)$ & $<0.001$ & $0.97(0.87-1.08)$ & 0.561 & $1.64(1.34-2.00)$ & $<0.001$ \\
\hline \multicolumn{9}{|l|}{ Household income } \\
\hline Poor & Ref & & Ref & & Ref & & Ref & \\
\hline Moderate & $0.81(0.59-1.11)$ & 0.183 & $0.7 \mid(0.40-1.27)$ & 0.246 & $0.84(0.61-1.15)$ & 0.274 & $0.7 \mid(0.40-1.28)$ & 0.258 \\
\hline Well-off & $0.73(0.53-1.00)$ & 0.052 & $0.73(0.40-1.33)$ & 0.310 & $0.78(0.57-1.08)$ & 0.139 & $0.75(0.4 \mathrm{I}-\mathrm{I} .36)$ & 0.336 \\
\hline Rich & $0.57(0.25-1.32)$ & 0.189 & $-\ddagger$ & $-\ddagger$ & $0.61(0.26-1.43)$ & 0.254 & $-\ddagger$ & $-\ddagger$ \\
\hline \multicolumn{9}{|l|}{ Hypertension } \\
\hline No & Ref & & Ref & & Ref & & Ref & \\
\hline Yes & I.7I (I.63-I.80) & $<0.001$ & $1.22(1.10-1.36)$ & $<0.001$ & $1.54(1.47-1.62)$ & $<0.001$ & $1.18(1.06-1.32)$ & 0.002 \\
\hline \multicolumn{9}{|l|}{ Hyperlipidemia } \\
\hline No & Ref & & Ref & & Ref & & Ref & \\
\hline Yes & $3.11(2.87-3.36)$ & $<0.001$ & $3.75(3.28-4.30)$ & $<0.001$ & $3.02(2.79-3.27)$ & $<0.001$ & $3.70(3.23-4.24)$ & $<0.001$ \\
\hline \multicolumn{9}{|l|}{$\mathrm{CHD}$} \\
\hline No & Ref & & Ref & & Ref & & Ref & \\
\hline Yes & $2.71(2.29-3.20)$ & $<0.001$ & $1.55(1.04-2.31)$ & 0.031 & $2.23(1.88-2.64)$ & $<0.001$ & $\mathrm{I} .48(\mathrm{I} .00-2.2 \mathrm{I})$ & 0.053 \\
\hline \multicolumn{9}{|l|}{ Stroke } \\
\hline No & Ref & & Ref & & Ref & & Ref & \\
\hline Yes & $3.01(2.55-3.56)$ & $<0.001$ & $1.22(0.77-1.94)$ & 0.392 & $2.49(2.10-2.96)$ & $<0.001$ & $1.16(0.73-1.84)$ & 0.534 \\
\hline \multicolumn{9}{|l|}{ Smoking } \\
\hline Non-smoker & Ref & & Ref & & Ref & & Ref & \\
\hline Ex-smoker & $1.20(0.95-1.33)$ & 0.191 & $1.15(0.83-1.61)$ & 0.409 & $1.14(0.95-1.37)$ & 0.169 & $0.98(0.69-1.39)$ & 0.887 \\
\hline Smoker & I.18 (I.09-I.27) & $<0.001$ & $1.10(0.94-1.29)$ & 0.254 & $1.13(1.03-1.24)$ & 0.010 & $0.91(0.75-1.09)$ & 0.307 \\
\hline \multicolumn{9}{|l|}{ Drinking } \\
\hline Non-drinkers & Ref & & Ref & & Ref & & Ref & \\
\hline Light & $0.99(0.93-1.06)$ & 0.843 & $0.99(0.87-1.12)$ & 0.854 & $1.00(0.94-1.07)$ & 0.937 & $0.98(0.86-I .12)$ & 0.777 \\
\hline Moderate & $0.97(0.91-1.03)$ & 0.298 & $0.99(0.87-1.14)$ & 0.936 & $0.97(0.90-1.03)$ & 0.296 & $0.99(0.86-1.14)$ & 0.912 \\
\hline Heavy & $0.94(0.86-1.02)$ & 0.126 & $1.26(1.11-1.47)$ & 0.003 & $0.73(0.66-0.80)$ & $<0.001$ & I.08 (0.9I-I.29) & 0.379 \\
\hline
\end{tabular}

(Continued) 
Table 3 (Continued).

\begin{tabular}{|c|c|c|c|c|c|c|c|c|}
\hline \multirow[t]{2}{*}{ Variables } & \multicolumn{4}{|c|}{ Crude OR $(95 \% \mathrm{Cl}) ; P$ value } & \multicolumn{4}{|c|}{ Adjusted OR $†(95 \% \mathrm{Cl}) ; P$ value } \\
\hline & T2DM & $\boldsymbol{P}$ & Prediabetes & $P$ & T2DM & $P$ & Prediabetes & $P$ \\
\hline \multicolumn{9}{|l|}{ Sleep duration (Hour) } \\
\hline$\leq 5$ & $1.55(\mid .42-1.70)$ & $<0.001$ & $1.30(1.08-1.56)$ & 0.005 & $1.44(1.32-1.58)$ & $<0.001$ & $1.29(1.07-1.55)$ & 0.007 \\
\hline $6-9$ & Ref & & Ref & & Ref & & Ref & \\
\hline$>9$ & $1.64(1.30-2.07)$ & $<0.001$ & $1.18(0.70-1.98)$ & 0.531 & $1.46(1.15-1.84)$ & 0.002 & $1.15(0.68-1.93)$ & 0.603 \\
\hline \multicolumn{9}{|l|}{ Family diabetes } \\
\hline No & Ref & & Ref & & Ref & & Ref & \\
\hline Yes & $3.97(3.67-4.30)$ & $<0.001$ & $2.72(2.3 \mid-3.21)$ & $<0.001$ & $4.68(4.3 I-5.08)$ & $<0.001$ & $2.85(2.4 I-3.36)$ & $<0.001$ \\
\hline \multicolumn{9}{|l|}{ Fish } \\
\hline No & Ref & & Ref & & Ref & & Ref & \\
\hline Yes & $0.75(0.65-0.87)$ & $<0.001$ & $0.59(0.42-0.82)$ & $<0.001$ & $0.77(0.67-0.90)$ & 0.001 & $0.59(0.43-0.83)$ & 0.002 \\
\hline \multicolumn{9}{|l|}{ Vegetables } \\
\hline$<100$ & Ref & & Ref & & Ref & & Ref & \\
\hline$\geq 100$ & $1.09(1.03-1.14)$ & 0.001 & $1.46(1.32-1.61)$ & $<0.001$ & $1.10(1.05-1.16)$ & $<0.001$ & $1.46(1.32-1.61)$ & $<0.001$ \\
\hline \multicolumn{9}{|l|}{ Soy } \\
\hline No & Ref & & Ref & & Ref & & Ref & \\
\hline Yes & $0.72(0.65-0.79)$ & $<0.001$ & $0.57(0.46-0.72)$ & $<0.001$ & $0.73(0.66-0.80)$ & $<0.001$ & $0.58(0.46-0.72)$ & $<0.001$ \\
\hline \multicolumn{9}{|l|}{ Dairy Product } \\
\hline No & Ref & & Ref & & Ref & & Ref & \\
\hline Yes & $1.23(1.17-1.30)$ & $<0.001$ & $0.80(0.7 I-0.90)$ & $<0.001$ & $1.17(1.11-1.24)$ & $<0.001$ & $0.79(0.7 \mid-0.89)$ & $<0.001$ \\
\hline \multicolumn{9}{|l|}{ Fruit } \\
\hline No & Ref & & Ref & & Ref & & Ref & \\
\hline Yes & $0.65(0.62-0.68)$ & $<0.001$ & $0.59(0.53-0.65)$ & $<0.001$ & $0.66(0.63-0.70)$ & $<0.001$ & $0.59(0.54-0.65)$ & $<0.001$ \\
\hline
\end{tabular}

Notes: $\nmid$ OR were adjusted for ther factors; $\ddagger$ The number of people in this group is 0 and cannot be brought into the formula to calculate the percentage. Abbreviation: OR, odds ratios.

limited to date. In this investigation focusing on the Nanchong region, the overall prevalence of T2DM was $13.9 \%$. To the best of our knowledge, this is the first largescale diabetes survey conducted for middle-aged and elderly adults in a third-tier city in western China. The T2DM prevalence in this study was higher than that in previous studies carried out in China. ${ }^{20,21}$ The higher prevalence in this region may be related to the ongoing urbanization and unique nutritional habits among the different provinces of China. ${ }^{22}$ Moreover, the higher average age of our study's target population, compared to other studies, might have been a critical factor underlying our results. Our findings highlight that age is an independent risk factor of T2DM in China. ${ }^{23}$ Interestingly, the health habits and dietary patterns of middle-aged and elderly people are better than those of young people, thereby reducing the incidence of diseases. In the current study, the incidence of prediabetes was only $3.7 \%$, which was lower than that previously reported in China. ${ }^{24-26}$
Since prediabetes was predominantly asymptomatic, people largely ignored routine blood glucose testing. This may be related to low awareness of prediabetes and insufficient attention to blood glucose in older adults. ${ }^{24-26}$ This part of the population was not included in our study due to the lack of blood glucose data. However, we will address this group and report on the prevalence of T2DM and prediabetes in this sector of the population in future work. It is noteworthy to mention that it is imperative to raise awareness on diabetes and stress the relevance of blood glucose testing, especially among middle-aged and older adults in western China, where the medical system requires improvements.

This study showed the association of BMI with T2DM and prediabetes. High BMI has been reported to be one of the strongest determinants of T2DM. ${ }^{27}$ Compared with other European and American nations, Chinese participants had a higher comparative risk of T2DM than participants of other ethnic groups, despite having the same 
BMI. ${ }^{28}$ The present study found that hypertension, hyperlipidemia, CHD, and stroke were positively related to T2DM, consistent with previous studies. ${ }^{29-31}$ Hyperlipidemia was also associated with prediabetes. Unfortunately, we were unable to determine whether the comorbidity was the cause or consequence of T2DM and prediabetes. However, it is worth noting that individuals with hypertension, hyperlipidemia, or CHD were at a higher risk of T2DM. Individuals with prediabetes and T2DM are at a higher risk of developing macrovascular complications, including hypertension, CHD, and stroke, ${ }^{32}$ and more than $60 \%$ of older adults with T2DM have at least one comorbidity. ${ }^{33}$ Therefore, T2DM of middle-aged and elderly people is a significant public health concern receiving widespread attention given its substantial morbidity, mortality, and economic burden.

Our data showed that compared with low-level education, persons with middle-level education had a lower risk of T2DM. A previous study showed that higher education was significantly related to better management of diabetes among older adults in China. ${ }^{34}$ Furthermore, higher education was positively associated with awareness and control of diabetes among the American elderly. ${ }^{35}$ This pattern could be explained because higher education is associated with better access and understanding of diabetes.

We found that current smokers have a significantly higher risk of developing T2DM than ex-smokers and nonsmokers. Helmut et al demonstrated that exposure to smoke and second-hand smoke strongly affects the risk of T2DM and the probability of its complications. ${ }^{36}$ Furthermore, given that more women than men smoke, a higher incidence of smoking-related diabetes in females is expected in the future. ${ }^{37}$ Our results show that drinking alcohol reduced the risk of T2DM, as alcohol intake $\geq 30$ $\mathrm{g} /$ day lowered the risk of T2DM after adjusting for other factors in the analysis. This is consistent with previous results showing that moderate alcohol consumption is associated with a lower risk of T2DM. Interestingly, for current non-drinkers and never-drinkers, those with alcohol intake $<63 \mathrm{~g} /$ day had a lower risk of T2DM, while the risk increased with higher alcohol amounts. ${ }^{38}$ However, this benefit might be limited to women only since men tended to have higher risks of T2DM even at very low levels of alcohol consumption. ${ }^{39}$

Some dietary factors, such as the intake of soy and fruit, were protective against T2DM and prediabetes, in line with previous research. ${ }^{40-43}$ In this study, eating dairy products was protective against prediabetes. However, it also increased the risk of T2DM, suggesting an inconclusive relationship between dairy products and diabetes. Our data support that the physiologic response to dairy products may differ according to the type of dairy product, the fat content, and the individual's age and health status. ${ }^{44}$ Thus, it cannot be simply concluded that dairy products, in general, reduce the risk of diabetes. ${ }^{45-47}$

Our results contrast with evidence supporting a beneficial effect of vegetable intake in preventing $\mathrm{T}_{2} \mathrm{DM}{ }^{42,48}$ In Chinese cuisine, vegetables are mainly stirfried in oil. ${ }^{49}$ In contrast, Europeans and Americans consume vegetables primarily in salads, boiled or steamed. ${ }^{50}$ Stir-frying involves a greater amount oil than salads and boiled dishes, with the average amount of oil being 12 $\mathrm{g}$ per $100 \mathrm{~g}$ of vegetables. ${ }^{49}$ This excessive oil in vegetable-based dishes is known to increase the risk of developing diabetes. ${ }^{51}$

This cross-sectional study is representative of the middle-aged population not only because it was conducted in eight communities of Nanchong but also because of its large sample size. Nevertheless, this study also has some limitations. First, the results were specific to the urban population. Future research should examine individuals from rural areas to confirm the results obtained in the present study. Second, although we also investigated the risk factors of prediabetes, the findings should be interpreted with caution, as a large number of individuals in this group had incomplete information and were excluded. The prevalence of T2DM and prediabetes in the excluded population should be investigated to further confirm our findings. Moreover, individual awareness of prediabetes needs to be assessed in future studies. Finally, we did not collect data on diabetes treatment and glycated hemoglobin levels and did not assess the impact of these factors on diabetes and prediabetes.

\section{Conclusion}

The prevalence of T2DM and prediabetes was $13.9 \%$ and $3.1 \%$, respectively, among middle-aged and older adults in Nanchong. Advanced age, high BMI, abdominal obesity, a family history of diabetes, hyperlipidemia, and smoking increased the risk of T2DM and prediabetes. Soy and fruit intake was beneficial in preventing T2DM and prediabetes, whereas vegetable intake increased the risk, mainly due to the large amount of oil used in the Chinese cooking method. These findings can assist the early identification of persons at high risk of T2DM and prediabetes and the implementation of timely lifestyle interventions to prevent these conditions. 


\section{Data Sharing Statement}

After publication, the authors intend to share individual de-identified participant data and all the clinical data collected from the patients. Besides, the statistical results and ethical approval consent are available for one year upon request through an e-mail:xishengx@126.com.

\section{Acknowledgments}

We thank all the participants of this study for their contribution. This work was supported by Merck \& Co., Inc. and the Community Comprehensive Management Program at the Nanchong Central Hospital (grant no. 20100167). The authors would like to thank the Comprehensive Management Center of Chronic Diseases of the Nanchong Central Hospital and the Community Service Centers in the Shunqing District, Nanchong. Mengdi Xia and Kaixiang Liu are co-first authors in this study.

\section{Disclosure}

The authors declare that the research was conducted in the absence of any commercial or financial relationships that could be construed as a potential conflict of interest.

\section{References}

1. IDF. International Diabetes Federation Diabetes Atlas. 9th ed. Brussels: IDF; 2019.

2. Chen M, Zhang X, Fang Q, Wang T, Li T, Qiao H. Three single nucleotide polymorphisms associated with type 2 diabetes mellitus in a Chinese population. Exp Ther Med. 2017;13(1):121-126.

3. Shah HS, Morieri ML, Marcovina SM, et al. Modulation of GLP-1 levels by a genetic variant that regulates the cardiovascular effects of intensive glycemic control in ACCORD. Diabetes Care. 2018;41 (2):348-355.

4. Feng W, Mao G, Li Q, et al. Effects of chromium malate on glycometabolism, glycometabolism-related enzyme levels and lipid metabolism in type 2 diabetic rats: a dose-response and curative effects study. J Diabetes Investig. 2015;6(4):396-407.

5. Poppe L, Crombez G, De Bourdeaudhuij I, Van der Mispel C, Shadid S, Verloigne M. Experiences and Opinions of Adults with Type 2 Diabetes Regarding a Self-Regulation-Based eHealth Intervention Targeting Physical Activity and Sedentary Behaviour. Int J Environ Res Public Health. 2018;15:5.

6. Tabák AG, Herder C, Rathmann W, Brunner EJ, Kivimäki M. Prediabetes: a high-risk state for diabetes development. The Lancet. 2012;379(9833):2279-2290.

7. Yang T, Jiang S, Barnett R, Peng S, Yu L. Individual and city-level determinants of secondhand smoke exposure in China. Int $J$ Health Geogr. 2015;14:1.

8. Zhang J, Jin J, Liu J, et al. A study of the correlation of insulin resistance and leptin with inflammatory factors and vascular endothelial injury in T2DM patients with CHD. Exp Ther Med. 2018;265-269.

9. Ma X, Wang Z, Liu X. Progress on catastrophic health expenditure in China: evidence from China Family Panel Studies (CFPS) 2010 to 2016. Int J Environ Res Public Health. 2019;16:23.
10. Roden M. Diabetes mellitus: definition, classification and diagnosis. Wien Klin Wochenschr. 2016;128(Suppl S2):S37-40. doi:10.1007/ s00508-015-0931-3

11. al. WECe. Appropriate body-mass index for Asian populations and its implications for policy and intervention strategies. The Lancet. 2004;363(9403):157-163.

12. Fan Y, Wang R, Ding L, et al. Waist circumference and its changes are more strongly associated with the risk of Type 2 diabetes than body mass index and changes in body weight in chinese adults. J Nutr. 2020.

13. Programme WToH. Guidelines for Controlling and Monitoring the Tobacco Epidemic. Geneva: World Health Organization [WHO; 1997.

14. Martínez-Larrad MT, Corbatón-Anchuelo A, Fernández-Pérez C, Lazcano-Redondo Y, Escobar-Jiménez F, Serrano-Ríos M. Metabolic syndrome, glucose tolerance categories and the cardiovascular risk in Spanish population. Diabetes Res Clin Pract. 2016;114:23-31.

15. Xiao J, Wu CL, Gao YX, et al. Prevalence of metabolic syndrome and its risk factors among rural adults in Nantong, China. Sci Rep. 2016;6:38089.

16. Lee PH, Macfarlane DJ, Lam TH, Stewart SM. Validity of the International Physical Activity Questionnaire Short Form (IPAQ-SF): a systematic review. Int $J$ Behav Nutr Phys Act. 2011;8:115.

17. Fan ML, He JP. Chinese guidelines for data processing and analysis concerning the International Physical.pdf. Zhonghua Liu Xing Bing Xиe Za Zhi. 2014;961-964.

18. Djbcf RI, Monk TH, Berman SR, Kupfer DJ. The Pittsburgh sleep quality index: a new instrument for psychiatric practice and research. Psychiatry Res. 1989;28:193-213.

19. Liu R, Li Y, Mao Z, et al. Gender-specific independent and combined dose-response association of napping and night sleep duration with type 2 diabetes mellitus in rural Chinese adults: the RuralDiab study. Sleep Med. 2018;45:106-113.

20. Jing Z, Chu J, Imam Syeda Z, et al. Catastrophic health expenditure among type 2 diabetes mellitus patients: a province-wide study in Shandong, China. J Diabetes Investig. 2019;10(2):283-289.

21. Song Y, Zhang X, Zhang H, et al. Prevalence of diabetes and prediabetes in adults from a Third-Tier City in Eastern China: a cross-sectional study. Diabetes Ther. 2019;10(4):1473-1485.

22. Zhu W, Chi A, Sun Y. Physical activity among older Chinese adults living in urban and rural areas: a review. J Sport Health Sci. 2016;5 (3):281-286.

23. Mao F, Zhu X, Liu S, et al. Age as an independent risk factor for diabetic peripheral neuropathy in chinese patients with type 2 diabetes. Aging Dis. 2019;10(3):592-600.

24. Wang WD, Xing L, Teng JR, Li S, Mi NA. Effects of basal insulin application on serum visfatin and adiponectin levels in type 2 diabetes. Exp Ther Med. 2015;9(6):2219-2224.

25. Xu Y, Wang $\mathrm{L}, \mathrm{He} \mathrm{J}$, et al. Prevalence and control of diabetes in Chinese adults. JAMA. 2013;310(9):948-959.

26. Menke A, Casagrande S, Geiss L, Cowie CC. Prevalence of and trends in diabetes among adults in the United States, 1988-2012. JAMA. 2015;314(10):1021-1029.

27. Zhang S, Lei R, Wu J, et al. Role of high mobility group A1 and body mass index in the prognosis of patients with breast cancer. Oncol Lett. 2017;14(5):5719-5726.

28. Zhang Y, Santosa A, Wang N, et al. Prevalence and the association of body mass index and other risk factors with prediabetes and type 2 diabetes among 50,867 adults in China and Sweden: a cross-sectional study. Diabetes Ther. 2019;10(6):2061-2077.

29. Mizukami H, Inaba W, Takahashi K, Inoue K, Sawanobori K, Yagihashi S. Augmented reduction of islet beta-cell mass in Goto-Kakizaki rats fed high-fat diet and its suppression by pitavastatin treatment. J Diabetes Investig. 2012;3(3):235-244. 
30. Teh WL, Abdin E, Vaingankar JA, et al. Prevalence of stroke, risk factors, disability and care needs in older adults in Singapore: results from the WiSE study. BMJ Open. 2018;8(3):e020285.

31. Cheng Q, Hu J, Yang P, et al. Sarcopenia is independently associated with diabetic foot disease. Sci Rep. 2017;7(1):8372.

32. Okwechime IO, Roberson S, Odoi A. Prevalence and predictors of pre-diabetes and diabetes among adults 18 years or older in Florida: a multinomial logistic modeling approach. PLoS One. 2015;10(12): e0145781.

33. Fong JH. Disability incidence and functional decline among older adults with major chronic diseases. BMC Geriatr. 2019;19(1):323.

34. Liu M, Wang J, He Y, et al. Awareness, treatment and control of type 2 diabetes among Chinese elderly and its changing trend for pas decade. BMC Public Health. 2016;16:278.

35. Dupre ME, Silberberg M, Willis JM, Feinglos MN. Education, glucose control, and mortality risks among U.S. older adults with diabetes. Diabetes Res Clin Pract. 2015;107(3):392-399.

36. Brath H, Kaser S, Tatschl C, Fasching P. Smoking, alcohol and diabetes (Update 2019). Wien Klin Wochenschr. 2019;131(Suppl 1):67-70

37. Kautzky-Willer A, Harreiter J, Sex PG. Gender differences in risk, pathophysiology and complications of type 2 diabetes mellitus. Endocr Rev. 2016;37(3):278-316.

38. Knott C, Bell S, Britton A. Alcohol consumption and the risk of type 2 diabetes: a systematic review and dose-response Meta-analysis of more than 1.9 million individuals from 38 observational studies. Diabetes Care. 2015;38:1804-1812.

39. Beulens JW, Rimm EB, Hu FB, Hendriks HF, Mukamal KJ. Alcohol consumption, mediating biomarkers, and risk of type 2 diabetes among middle-aged women. Diabetes Care. 2008;31(10):2050-2055.

40. Afshin A, Micha R, Khatibzadeh S, Mozaffarian D. Consumption of nuts and legumes and risk of incident ischemic heart disease, stroke, and diabetes: a systematic review and meta-analysis. Am J Clin Nutr. 2014;100(1):278-288.

41. Basiak-Rasala A, Rozanska D, Zatonska K. Food groups in dietary prevention of type 2 diabetes. Rocz Panstw Zakl Hig. 2019;70 (4):347-357.
42. Safabakhsh M, Koohdani F, Bagheri F, Siassi F, Khajehnasiri F, Sotoudeh G. Fruit and vegetable intake and pre-diabetes: a case-control study. Eur J Nutr. 2018;57(8):2953-2962.

43. Tang J, Wan Y, Zhao M, Zhong H, Zheng JS, Feng F. Legume and soy intake and risk of type 2 diabetes: a systematic review and meta-analysis of prospective cohort studies. Am J Clin Nutr. 2020;111(3):677-688.

44. Oc S, et al. Impact of a high intake of dairy product on insulin sensitivity in hyperinsulinemic adults: a crossover randomized controlled trial. Current Developments in Nutrition. 2017;3(8):nzz083.

45. Gijsbers L, Ding EL, Malik VS, de Goede J, Geleijnse JM, Soedamah-Muthu SS. Consumption of dairy foods and diabetes incidence: a dose-response meta-analysis of observational studies. Am J Clin Nutr. 2016;103(4):1111-1124.

46. Eussen SJ, van Dongen MC, Wijckmans N, et al. Consumption of dairy foods in relation to impaired glucose metabolism and type 2 diabetes mellitus: the Maastricht Study. Br J Nutr. 2016;115 (8):1453-1461.

47. Aune D, Norat T, Romundstad P, Vatten LJ. Dairy products and the risk of type 2 diabetes: a systematic review and dose-response meta-analysis of cohort studies. Am J Clin Nutr. 2013;98 (4):1066-1083.

48. Li M, Fan Y, Zhang X, Hou W, Tang Z. Fruit and vegetable intake and risk of type 2 diabetes mellitus: meta-analysis of prospective cohort studies. BMJ Open. 2014;4(11):e005497.

49. Pu G, Zheng M, Lu S, Huang J. Study on the Use of Cooking Oil in Chinese Dishes. Int J Environ Res Public Health. 2019;16:18.

50. Mamluk L, O’Doherty MG, Orfanos P, et al. Fruit and vegetable intake and risk of incident of type 2 diabetes: results from the consortium on health and ageing network of cohorts in Europe and the United States (CHANCES). Eur J Clin Nutr. 2017;71(1):83-91.

51. Zhuang P, Mao L, Wu F, Wang J, Jiao J, Zhang Y. Cooking oil consumption is positively associated with risk of Type 2 diabetes in a chinese nationwide cohort study. J Nutr. 2020.

Diabetes, Metabolic Syndrome and Obesity: Targets and Therapy

Dovepress

\section{Publish your work in this journal}

Diabetes, Metabolic Syndrome and Obesity: Targets and Therapy is an international, peer-reviewed open-access journal committed to the rapid publication of the latest laboratory and clinical findings in the fields of diabetes, metabolic syndrome and obesity research. Original research, review, case reports, hypothesis formation, expert opinion and commentaries are all considered for publication. The manuscript management system is completely online and includes a very quick and fair peer-review system, which is all easy to use. Visit http://www.dovepress.com/testimonials.php to read real quotes from published authors.

Submit your manuscript here: https://www.dovepress.com/diabetes-metabolic-syndrome-and-obesity-targets-and-therapy-journal 